\title{
Identification of genes and pathways involved in malignant pleural mesothelioma using bioinformatics methods
}

\author{
Xingsheng Liu, Kun Qian, Gaojun Lu, Peng Chen and Yi Zhang ${ }^{*}$ (D)
}

\begin{abstract}
Background: Malignant pleural mesothelioma (MPM) is a rare tumor in the pleura. This study was carried out to identify key genes and pathways that may be involved in MPM.

Methods: Microarray datasets GSE51024 and GSE2549 were analyzed for differentially expressed genes (DEGs) between normal and MPM tissues. The identified DEGs were subjected to functional analyses using bioinformatics tools.

Results: A total of 276 DEGs were identified, consisting of 187 downregulated and 79 upregulated genes. Gene ontology and Kyoto encyclopedia of genes and genomes pathway enrichment analysis indicated that the DEGs were enriched in extracellular structure organization, extracellular matrix, and ECM-receptor interaction. Due to high degree of connectivity among 24 hub genes, EZH2 and HMMR are likely to play roles in the carcinogenesis and progression of MPM. The two genes were found over-expressed in MPM tissues. Patients with elevated EZH2 and HMMR expressions had poor overall survival.
\end{abstract}

Conclusions: $\mathrm{EZH} 2$ and HMMR are identified to be the hub genes for MPM and they may be further characterized to better understand the molecular mechanisms underlying the carcinogenesis of MPM.

Keywords: Microarray, Gene expression profile, Cancer, Differentially expressed genes, Protein-protein interaction, Bioinformatics

\section{Background}

Malignant pleural mesothelioma (MPM) is a rare and highly lethal neoplasm arising from the mesothelial cells lining the pleural cavity [1]. However, due to wide use of asbestos, its incidence has been dramatically increasing since the mid-twentieth century [2]. Among histological types (epithelial, sarcomatoid, and biphasic or mixed), the sarcomatoid carcinoma is particularly associated with a more aggressive progression [3]. Because of a lack of effective treatment of the disease, overall survival (OS)

*Correspondence: zhangyi@xwhosp.org

Department of Thoracic Surgery, Xuanwu Hospital, Capital Medical

University, 45 Changchun Street, Beijing 100053, China of patients with MPM is around one year after diagnosis and up to two years after receiving intensive multimodality therapy [4]. Therefore, it is important to elucidate the molecular mechanisms underlying the carcinogenesis and progression of MPM.

During the past decade, tremendous progress has been made in elucidating the pathogenesis of MPM. For example, microarray technology has been used to examine the oncogenic genetic alterations in MPM, such as homozygous deletion of the 9p21 locus which harbors the p16/ CDNK2A gene, germline mutations of BAP1 and mutations of multiple Hippo genes [5, 6]. Moreover, NAT2, a polymorphic gene encoding enzymes in xenobiotic and oxidative metabolism or involved in genome stability, is 
found to increase MPM risk in asbestos-exposed populations [7]. From epigenetic perspectives, SETD2 (SET domain containing protein 2, a histone methyltransferase), specifically H3K36me3 (histone H3 trimethylated at lysine 36), is identified as epigenetic regulator of gene expression associated with MPM [5]. Based on the methylation status of the human androgen receptor gene, MPM was found to have a polyclonal origin [8]. An exploratory analysis proposed that PI3K/Akt/ mTOR signaling pathways and downstream proteins are frequently activated in MPM and can be used to provide prognostic information [9]. In addition, chronic inflammation also plays a key role in the pathogenesis of MPM as a result of asbestos exposure [10]. However, the moclular mechanisms underlying the disease are still largely unclear and more genes need to be identified to advance our understanding.

In the present study, two mRNA microarray datasets from Gene Expression Omnibus (GEO) databases were analyzed to identify the common DEGs between MPM and non-cancerous tissues. Enrichment analysis, network-based approaches and ONCOMINE data mining were applied to identify hub genes related to MPM. The findings would provide new insights into moclular mechanisms related to MPM pathogenesis and clues to develop diagnostic and therapeutic approaches for the caner.

\section{Materials and methods Microarray data}

The mRNA expression profiles were obtained from the GSE2549 and GSE51024 datasets [11]. The GSE2549 dataset contains $40 \mathrm{MPM}$ samples and 4 non-cancerous tissue samples. The GSE51024 dataset has 55 MPM samples and 41 non-cancerous tissue samples (Table 1). The two datasets were downloaded from GEO (http:// www.ncbi.nlm.nih.gov/geo) which is a public repository containing data obtained through high throughout gene expression assays, chips and microarrays. The data were uniformly pre-processed using the Robust Multichip Average algorithm for background correction, quantile normalization and log2-transformation [12]. The probes were converted into corresponding genes using the annotation information available from the gene platforms (GPL96-57554 and GPL570-55999, respectively.).
Identification of differentially expressed genes (DEGs)

The DEGs between MPM and non-cancerous tissue samples were identified using the limma package in $\mathrm{R}$ language. Adjusted P-values (adj. P) and false discovery rate were used to balance statistically significant genes and false-positives. The threshold of adj. P was set at $<0.05$ and $\mid \log 2$ (fold-change) $\mid>1$. Volcano figures were plotted to identify the DEGs in the two datasets using ggplot2 package (version 3.5.3). Venn diagram was then constructed to determine the common DEGs originated from both datasets.

Construction of protein-protein interaction (PPI) network, module analysis and hub gene selection

The Search Tool for the Retrieval of Interacting Genes (STRING; http://string-db.org) (version 10.0) provides uniquely comprehensive interaction information of experimental and predicted proteins. In this study, the PPI network of common DEGs was constructed using STRING online database. A combined score $>0.4$ was considered as the reliability threshold for interaction. Cytoscape (version 3.4.0; www.cytoscape.org), an open source bioinformatics software platform, was used to visualize the molecular interactions in the PPI networks obtained with the STRING online databases. Subsequently, the module analysis of the PPI network was preformed using Molecular Complex Detection (MCODE) (version 1.5.1), which is an APP for analyzing hub genes according to topology to find highly connected regions which had MCODE scores $>5$, degree cut-off $=2$, node score cut-off $=0.2$, Max depth $=100$ and $\mathrm{k}$-score $=2$. Then, the hub genes were selected from the module based on both the degree of the connectivity and the node status.

\section{Gene ontology (GO) and Kyoto encyclopedia of genes and genomes (KEGG) pathway enrichment analysis}

GO is widely used to annotate genes and gene products, and to functionally characterize high-throughput genome or transcriptome data. KEGG are databases capable of interacting with genomes, biological pathways, diseases, drugs, and chemicals [13, 14]. GO and KEGG pathway enrichment analyses were carried out using clusterProfiler package and Signaling Pathway Impact Analysis (SPIA) (v3.4.0). The threshold was set

Table 1 Details of MPM mRNA expression data collected from GEO data sets

\begin{tabular}{llllll}
\hline Author (year) & Materials & Accession/ID & Platform & Normal samples & MPM samples \\
\hline & Tissue & GSE2549 & GPL96-57554 & 4 & 40 \\
Suraokar et al. [1 1] & Tissue & GSE74190 & GPL570-55999 & 41 & 55
\end{tabular}

GEO Gene Expression Omnibus, GPL GEO platform, MPM Malignant Pleural Mesothelioma, mRNA messenger RNA 
at $P$-value $<0.05$. Enriched GO terms or KEGG signaling pathways were ranked based on the degree of connectivity of hub genes using GOplot package (version 1.4.0).

\section{Validation of the selected hub genes in bioinformatic database}

Oncomine (www.oncomine.org; Ion Torrent; Thermo Fisher Scientific, Inc.) is an online cancer microarray database developed to facilitate the discovery of oncogenes through genome-wide expression analyses [15]. To validate the expression level of the identified hub genes in MPM, gene expression data in the Oncomine database were analyzed with $P$-value set to $<0.05$, thresholds for fold-change and gene rank set to 'all'. In order to calculate the prognostic significance of the selected hub genes, Kaplan-Meier survival analyses were performed based on the clinical information from TCGA datasets using UALCAN, which is a comprehensive, user-friendly, and interactive web resource [16]. For this analysis, the patients were separated into high and low expression groups according to the median of the hub gene expression levels.

\section{Results}

\section{Identification of DEGs}

After standardization of the microarray results, a total of 827 and 1062 DEGs were identified in the GSE51024 and GSE2549 datasets, respectively. The volcano figures were ploted to visualize DEGs between MPM and noncancerous tissue samples (Fig. 1a, b). The Venn diagram
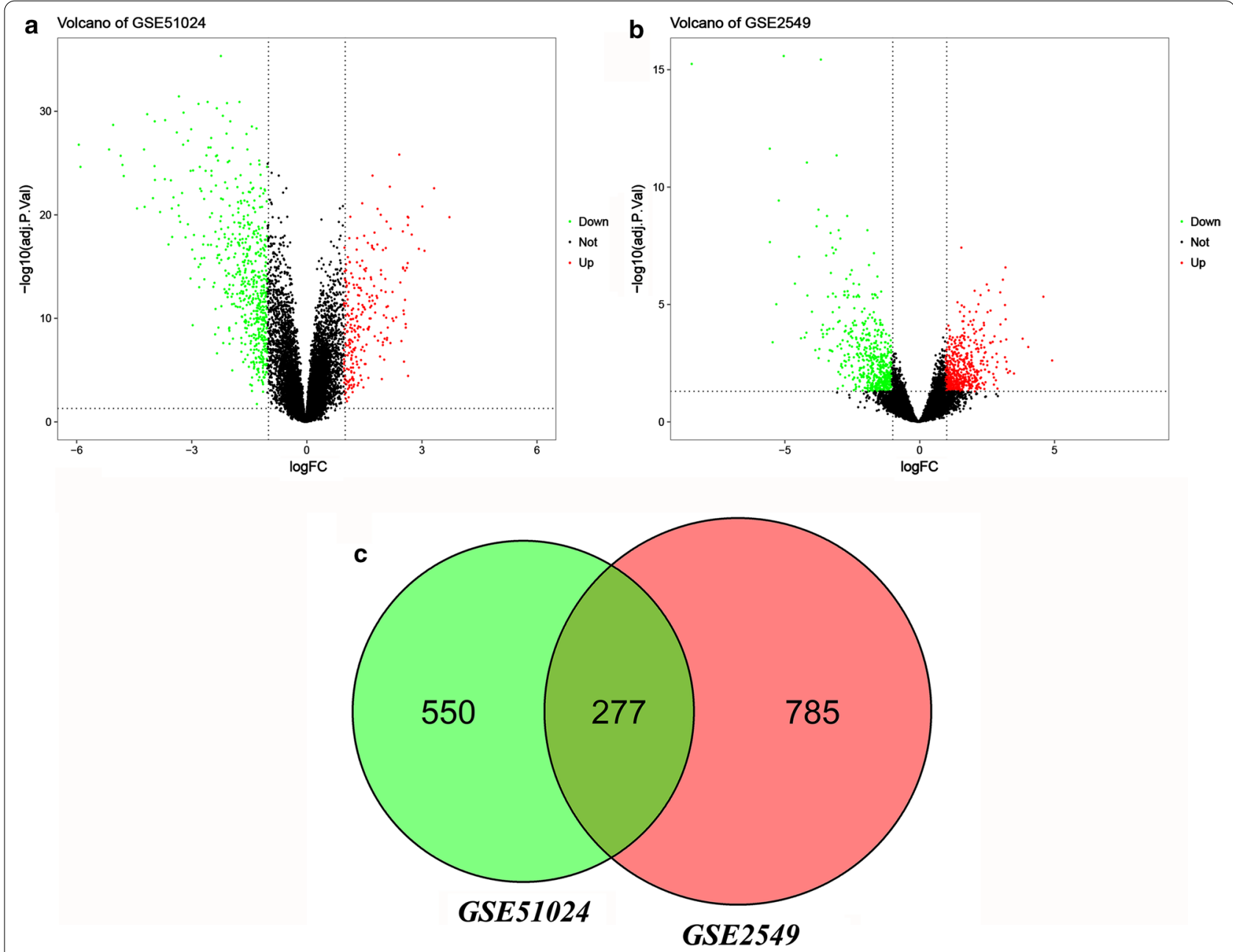

Fig. 1 Volcano plot and Venn diagram of DEGs in MPM mRNA expression profiling datasets. Volcano plots of the gene expression data from the a GSE50124, b GSE2549 datasets. DEGs were selected by P $<0.05$ and $\mid \log 2$ (fold-change)|> 1.The horizontal axis represents the log2 (fold change) between MPM samples and non-cancerous tissue samples; the vertical axis represents the -log10 (adjusted P-values). The red dots represent the co-upregulated DEGs and the blue dots represent the co-downregulated DEGs. c The grey overlap represents the common DEGs between the two datasets. DEGs differentially expressed genes 
showed that 276 DEGs were common in the two datasets, of which 187 and 79 genes were downregulated and upregulated in both datasets, respectively (Fig. 1c).

\section{Construction of PPI network and module analysis}

To investigate protein interactions, PPI network of the common DEGs was downloaded from STRING and the PPI network was constructed using Cytoscape. The network contained 239 nodes and 56,882 edges after removing isolated nodes (Fig. 2). And then, a module of significant hub genes was obtained from the PPI network of DEGs using MCODE, consisting of 24 nodes and 552 edges (Fig. 3).The hub genes were obtained with a connectivity degree of $\geq 19$ in the module. Considering the connectivity and the node status together, EZH2 and HMMR might play the most important roles in the carcinogenesis or progression of MPM (Table 2).

\section{GO and KEGG pathway enrichment analysis}

After GO and KEGG pathway enrichment analysis, the top 10 terms of each category ranked by adjusted $P$-value are presented in Fig. $4 \mathrm{a}$. These results showed

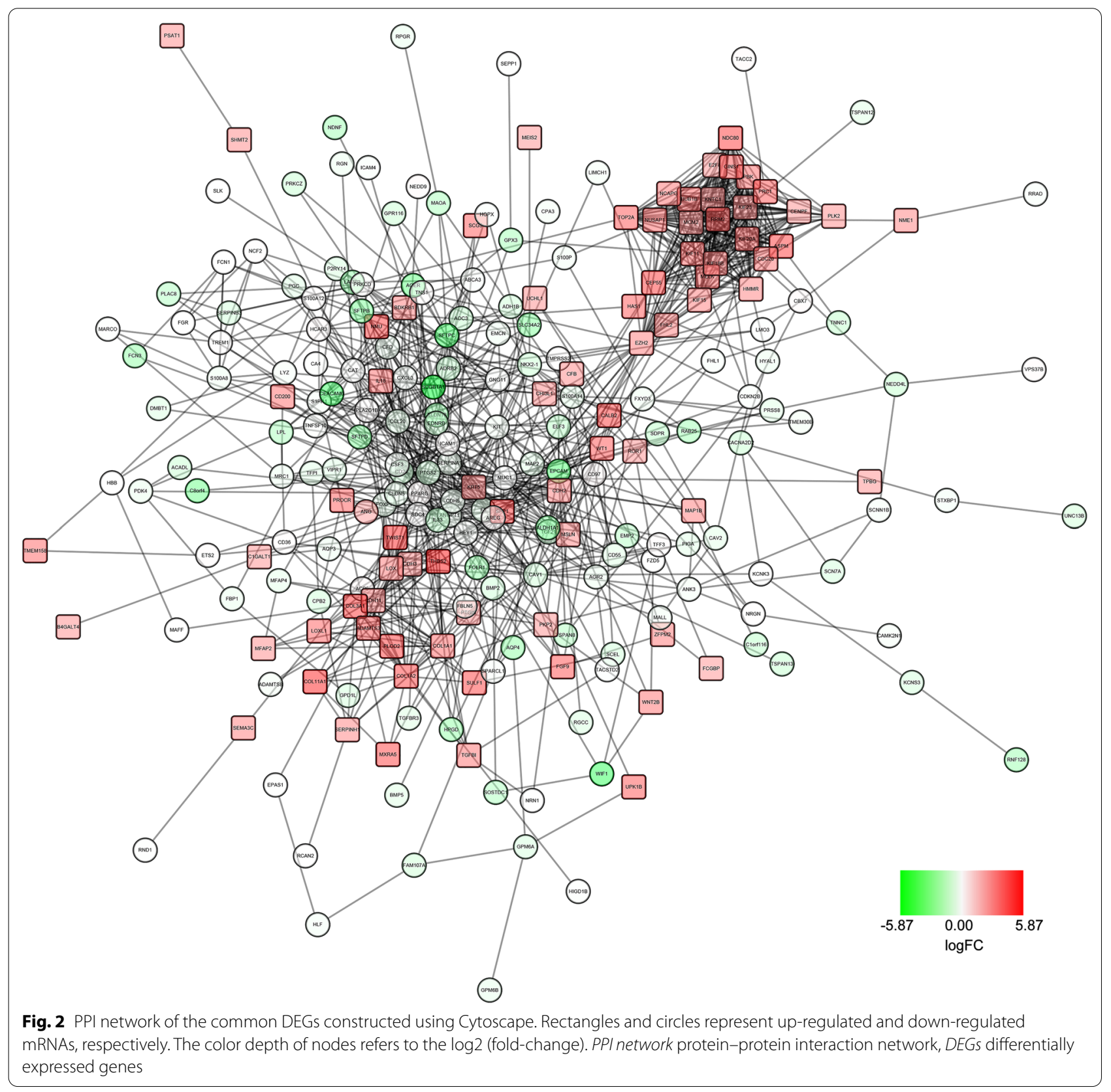




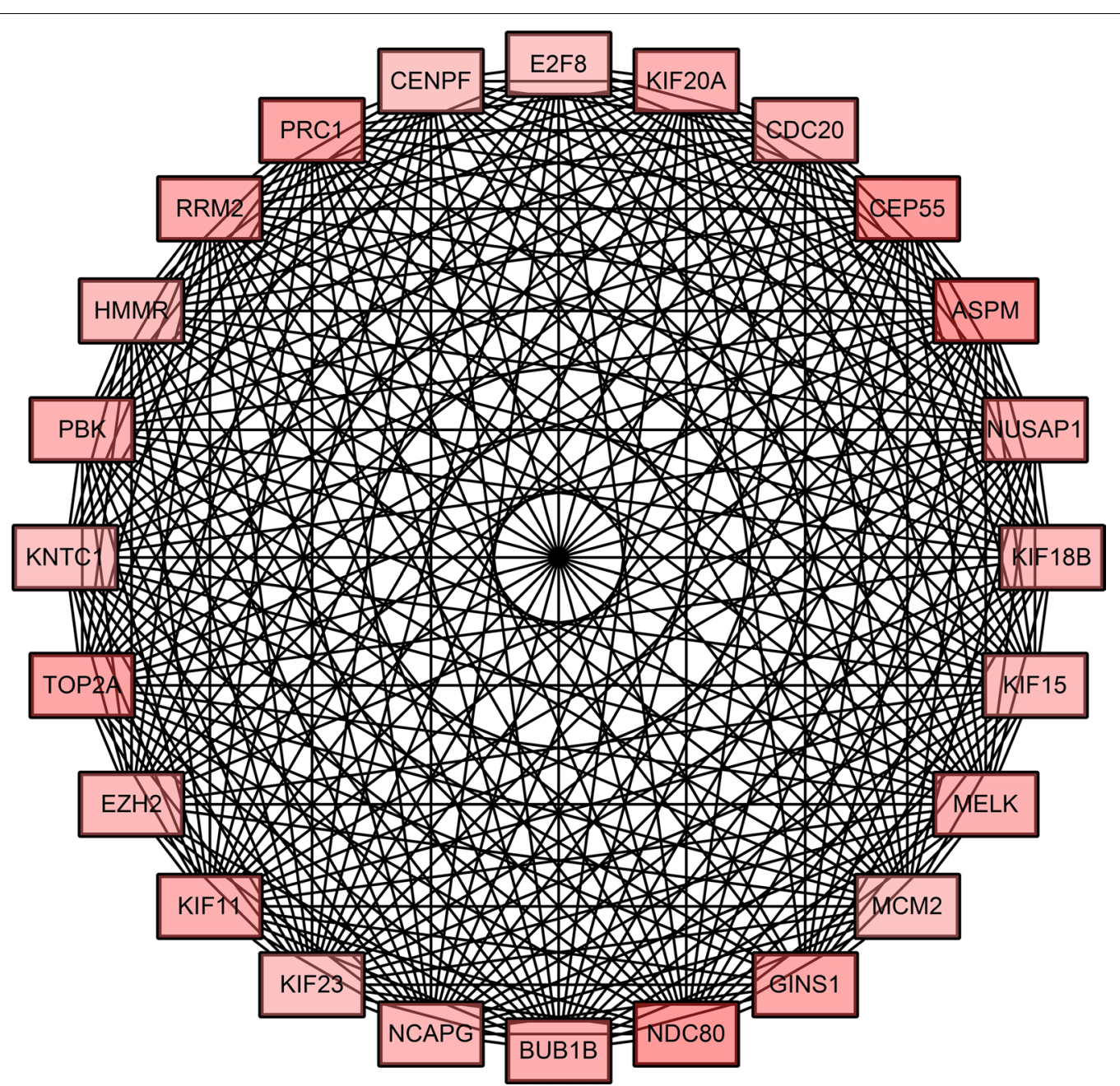

Fig. 3 A significant module of hub genes obtained from the PPI network of DEGs using MCODE. PPI network protein-protein interaction network, MCODE molecular complex detection

that the DEGs in biological process (BP) were mainly enriched in extracellular matrix organization, leukocyte migration, extracellular matrix organization, reproductive structure development, mesenchyme development, transmembrane receptor protein serine/ threonine kinase signaling pathway, renal system development, mesenchymal cell differentiation, regulation of cell-substrate adhesion and extracellular matrix assembly. The DEGs in cellular component (CC) were mainly enriched in extracellular matrix, collagen - containing extracellular matrix, membrane raft, membrane microdomain, membrane region, apical plasma membrane, collagen trimer, extracellular matrix component, fibrilar collagen trimer, and banded collagen fibril, while the DEGs in molecular function (MF) were significantly enriched in extracellular matrix structural constituent, channel regulator activity, cargo receptor activity, ion channel regulator activity, integrin binding, scavenger receptor activity, potassium channel regulator activity, signaling pattern recognition receptor activity, pattern recognition receptor activity, plateletderived growth factor binding. KEGG pathway analysis revealed that the the common DEGs were mainly enriched in Complement and coagulation cascades, AGE-RAGE signaling pathway in diabetic complications, ECM-receptor interaction and Malaria (Fig. 4b). The distribution of the common hub genes in the GO and KEGG pathway analysis is shown in Fig. 5 . The hub genes in BP, CC and MF were mainly enriched in extracellular structure organization (Fig. 5a), extracellular matrix (Fig. 5b) and extracellular matrix structural constituent (Fig. 5c), respectively. In the KEGG pathways the hub genes were mainly located in ECM - receptor interaction (Fig. 5d). 
Table 2 Characteristics of 24 hub genes obtained from a significant module of the PPI network

\begin{tabular}{|c|c|c|c|c|}
\hline Name & Full name & Degree & Regulation & MCODE node status \\
\hline $\mathrm{EZH} 2$ & Enhancer Of Zeste 2 Polycomb Repressive Complex 2 Subunit & 35 & Up & Clustered \\
\hline KIF11 & Kinesin Family Member 11 & 27 & Up & Clustered \\
\hline ASPM & Abnormal Spindle Microtubule Assembly & 26 & Up & Clustered \\
\hline HMMR & Hyaluronan Mediated Motility Receptor & 25 & Up & Seed \\
\hline CEP55 & Centrosomal Protein 55 & 25 & Up & Clustered \\
\hline CDC20 & Cell Division Cycle 20 & 25 & Up & Clustered \\
\hline$B \cup B 1 B$ & BUB1 Mitotic Checkpoint Serine/Threonine Kinase B & 25 & Up & Clustered \\
\hline PRC1 & Protein Regulator Of Cytokinesis 1 & 25 & Up & Clustered \\
\hline RRM2 & Ribonucleotide Reductase Regulatory Subunit M2 & 24 & Up & Clustered \\
\hline TOP2A & DNA Topoisomerase II Alpha & 24 & Up & Clustered \\
\hline PBK & PDZ Binding Kinase & 24 & Up & Clustered \\
\hline KIF20A & Kinesin Family Member 20A & 24 & Up & Clustered \\
\hline MCM2 & Minichromosome Maintenance Complex Component 2 & 24 & Up & Clustered \\
\hline KIF23 & Kinesin Family Member 23 & 24 & Up & Clustered \\
\hline NUSAP1 & Nucleolar And Spindle Associated Protein 1 & 24 & Up & Clustered \\
\hline NCAPG & Non-SMC Condensin I Complex Subunit G & 24 & Up & Clustered \\
\hline KIF15 & Kinesin Family Member 15 & 23 & Up & Clustered \\
\hline MELK & Maternal Embryonic Leucine Zipper Kinase & 23 & Up & Clustered \\
\hline NDC80 & NDC80 Kinetochore Complex Component & 23 & Up & Clustered \\
\hline CENPF & Centromere Protein F & 22 & Up & Clustered \\
\hline KNTC1 & Kinetochore Associated 1 & 22 & Up & Clustered \\
\hline KIF18B & Kinesin Family Member 18B & 21 & Up & Clustered \\
\hline E2F8 & E2F Transcription Factor 8 & 21 & Up & Clustered \\
\hline GINS1 & GINS Complex Subunit 1 & 19 & Up & Clustered \\
\hline
\end{tabular}

MCODE node status Molecular Complex Detection Node Status

\section{Validation of EZH2 and HMMR expression and their prognostic significance}

To validate the expression levels of EZH2 and $H M M R$, expression data from ONCOMINE were compared. The results showed that they were signifcantly up-regulated in MPM compared to normal tissues (Fig. 6a, b). To investigate the prognostic significance, Kaplan-Meier survival analysis was preformed. The log-rank test confrmed that patients with high levels of EZH2 and HMMR had significantly lower survival rate than those with low levels using UALCAN resource (Fig. 7).

\section{Discussion}

To identify new genes potentially related to MPM, we analyzed two mRNA microarray datasets. A total of 276 common DEGs between MPM tissues and non-cancerous tissues were identifed in the 2 datasets, including 187 downregulated genes and 79 upregulated genes. GO and pathway analyses were performed to explore functions of the DEGs. They were found enriched in extracellular structure organization, extracellular matrix, matrix structural constituent, and ECM-receptor interaction. EZH2 and HMMR were found to be related to MPM based on PPI analysis.

Previous studies have reported that overexpression of HAPLN1, one of the ECM proteins, increases tumorigenic properties of mesothelioma [17]. In addition, high expression of tenascin-C protein, an extracellular matrix glycoprotein known to have anti-adhesive characteristics in MPM, might play a role in invasive growth of MPM [18]. KEGG pathway analysis showed that the common DEGs are associated with complement and coagulation cascades, AGE-RAGE signaling pathway in diabetic complications, ECM-receptor interaction, and HMMR was riched in ECM-receptor interaction. Based on parallel sequencing, Hylebos et al. [19] found that tumor protein p53/DNA repair, cell cycle, mitogen-activated protein kinase, and phosphoinisitide 3-kinase (PI3K)/ AKT pathways are related to MPM. Furthermore, Cedrés et al. proposed that pS6 might be an independent prognostic factor in MPM [9]. Targeting this pathway could be of therapeutic significance. For instance, in the SWOG S0722 trial mTOR inhibitor everolimus (RAD001) has been demostrated to have limited clinical activity in advanced MPM patients [20]. 


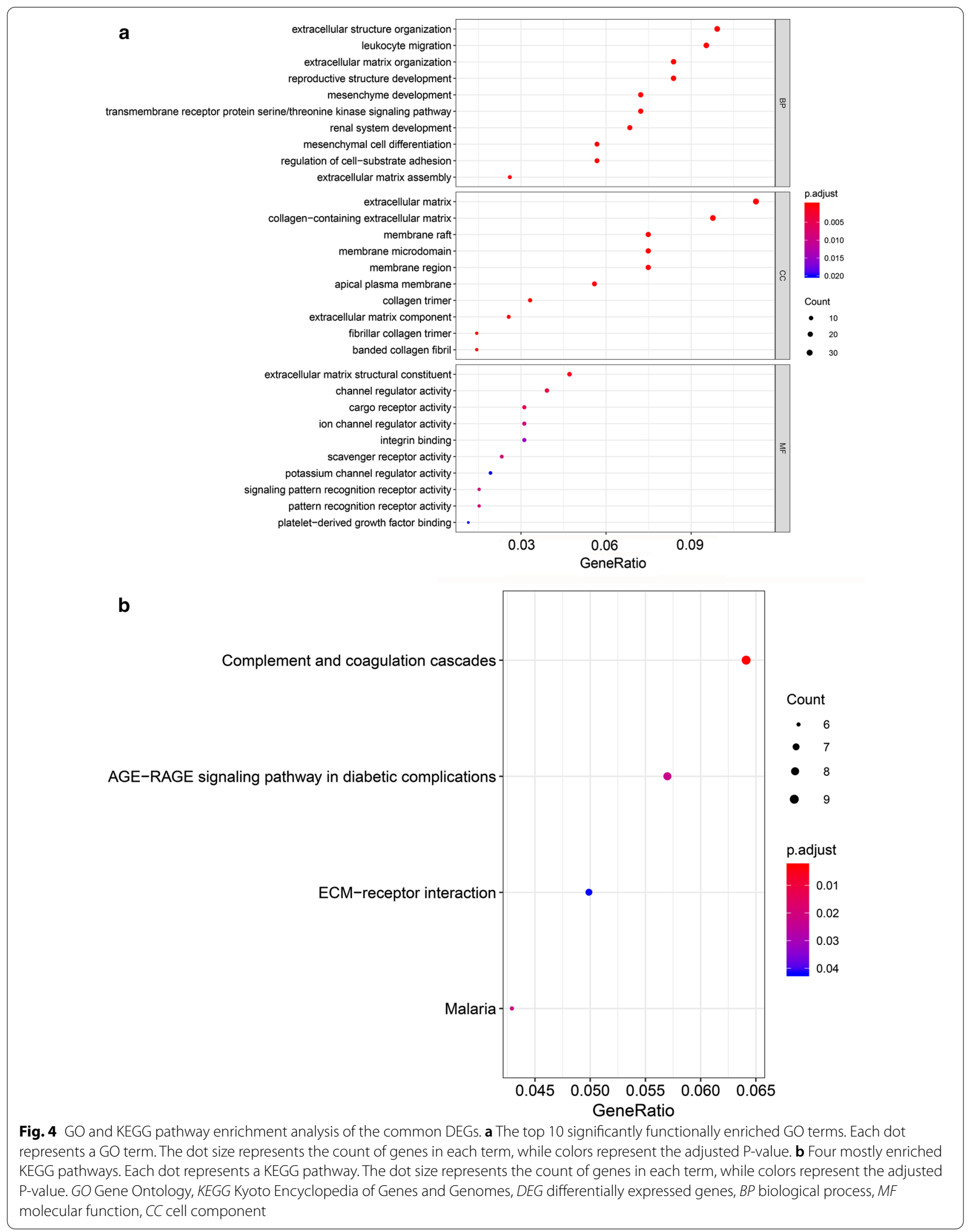




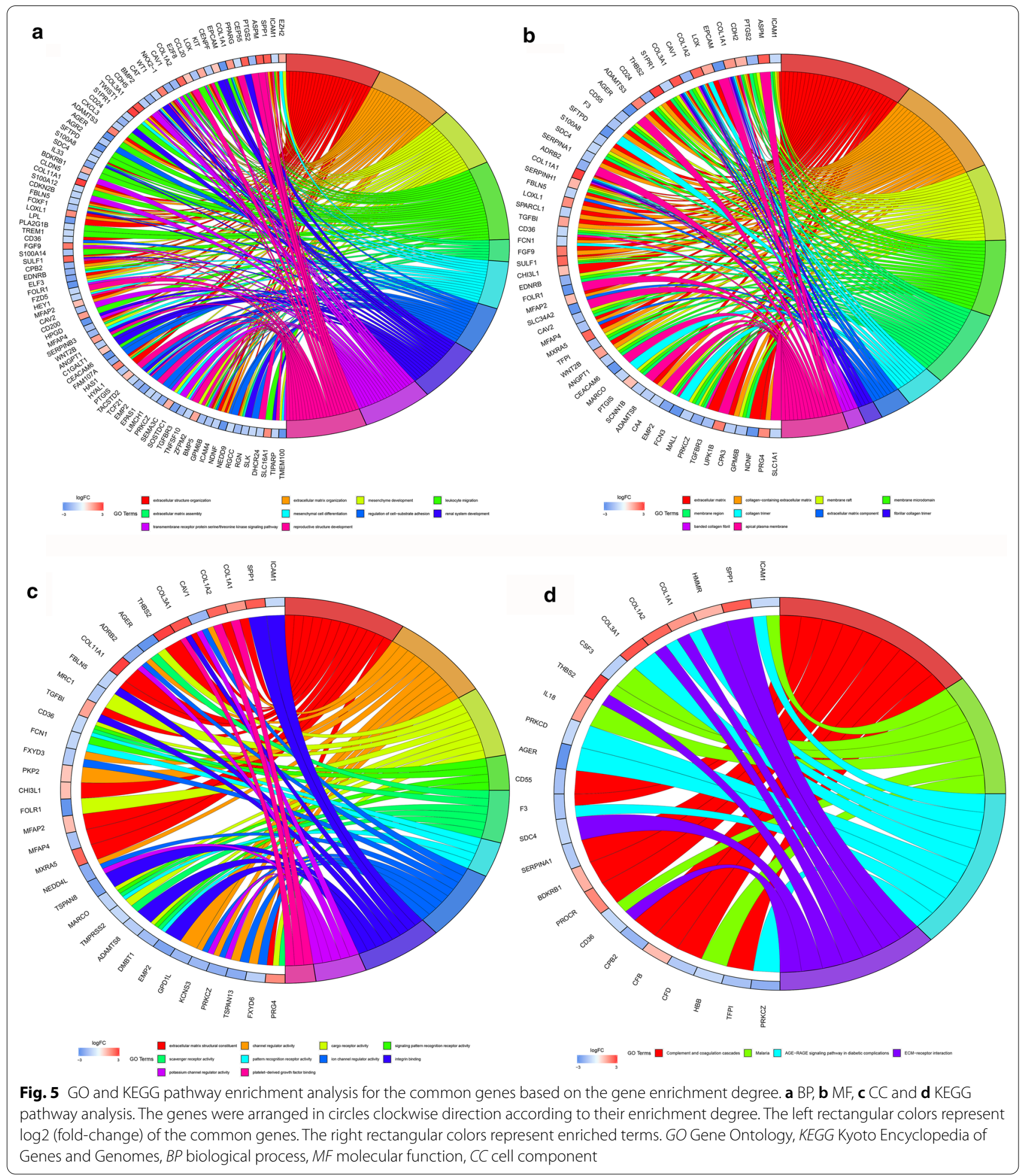

To examine the associations of protein functions of the identified 276 common DEGs, a PPI network was constructed, 24 DEGs were selected as hub genes because of their high degree of connectivity (>19) using MCODE in Cytoscape. According to the connectivity degree and the node status, EZH2 and HMMR have signifcant differences between MPM and non-cancerous tissue samples. EZH2 encodes a member of the Polycomb-group 

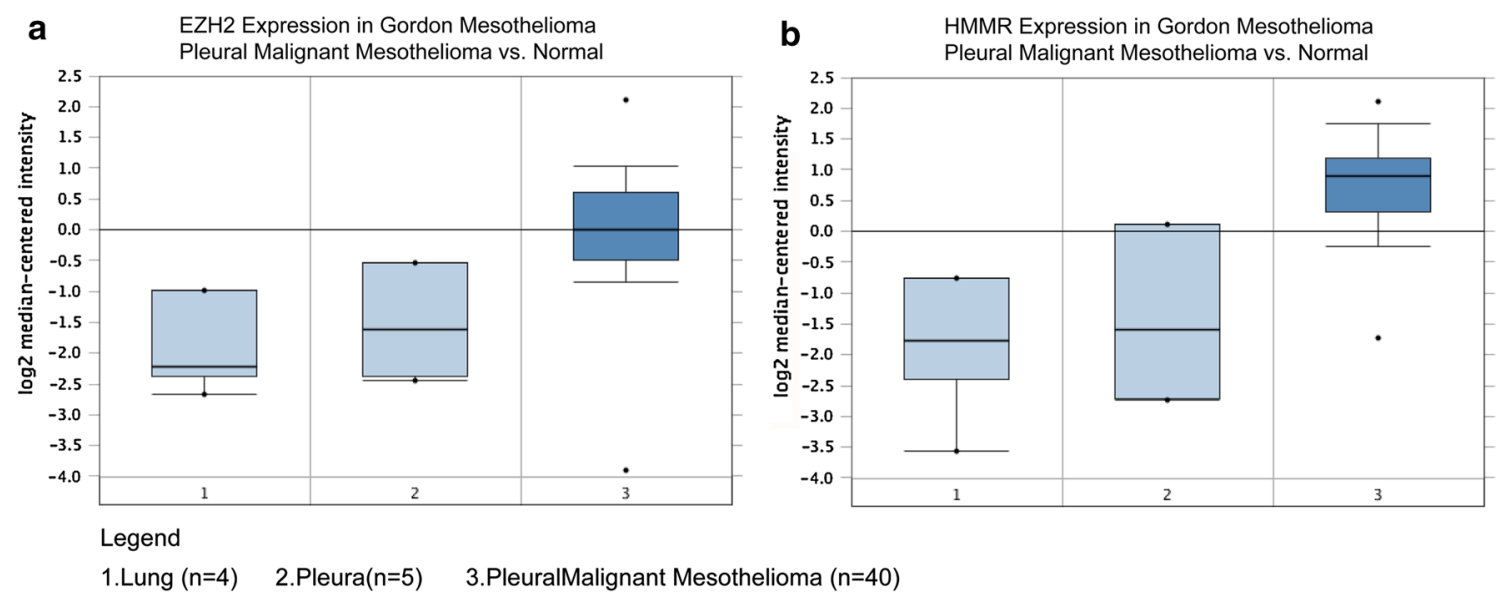

Fig. 6 mRNA expression levels of EZH2 (a) and HMMR (b) in MPM, lung and pleura tissue samples in the ONCOMINE database
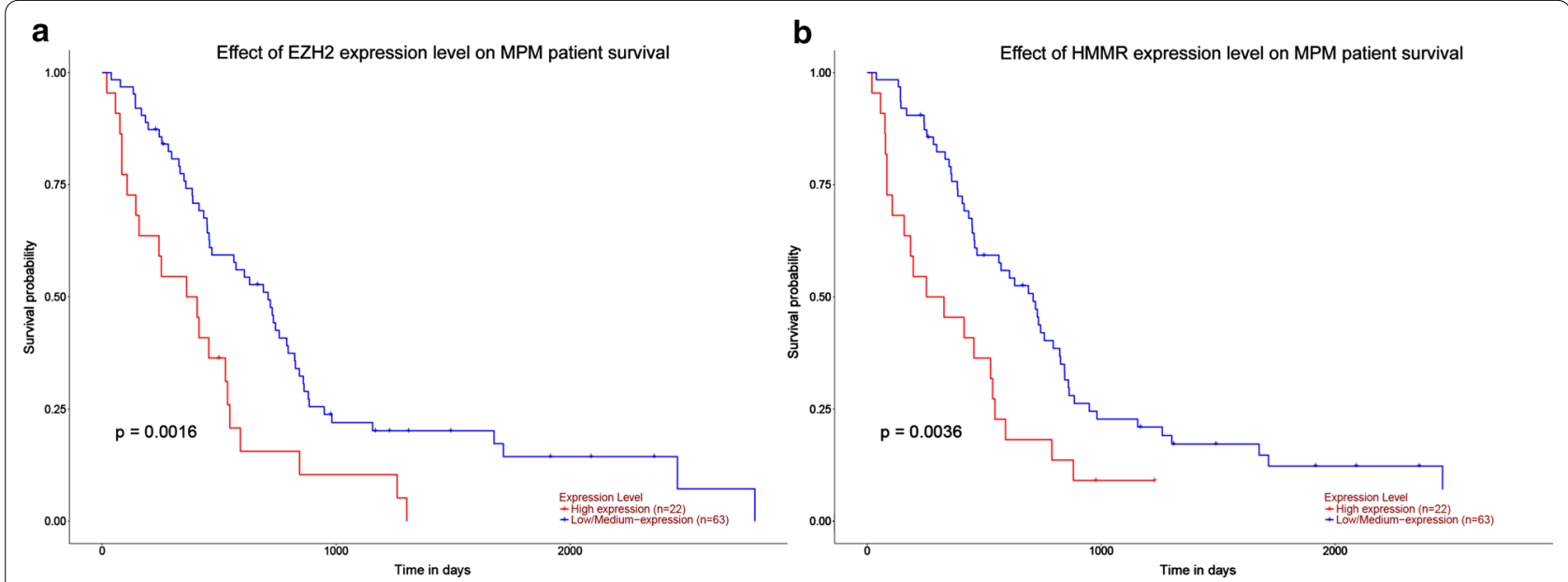

Fig. 7 UALCAN overall survival analysis of MPM patients with high and low expression of EZH2 (a) and HMMR (b)

(PcG) family in which the two main families are polycomb repressive complex 1 (PRC1) and PRC2 [21]. They are associated with the embryonic ectoderm development protein, the VAV1 oncoprotein, and the X-linked nuclear protein and may play a role in the hematopoietic and central nervous syste [22]. As previously reported, $\mathrm{EZH} 2$ is overexpressed in Weaver syndrome and primary cutaneous follicle center lymphoma [22, 23]. LaFave et al. [24] reported that loss of BAP1 promotes MPM cell proliferation by upregulating EZH2. The epigenetic gene H3K27me3 catalyzed by EZH2 is repressive which is aberrantly expressed during malignancy transformation in MPM [25]. Suppression of EZH2 using RNA interference was found to decrease the cancerogeneity of malignant mesothelioma cells [25]. In clinic practice, highly expressed EZH2 and deletion of BAP1 and MTAP can be detected by immunohistochemistry and may be used to distingush MPM from mesothelial hyperplasia [21]. HMMR (Hyaluronan Mediated Motility Receptor) is a protein coding gene influencing in cell motility [26]. The protein is detected in breast tissue and complexes with BRCA1 and BRCA2. And thus, HMMR potentially increases the risk of breast cancer. In addition, EZH2 and HMMR were also overexpressed in MPM from ONCOMINE and discovered to be associated with low overall survival in MPM patients, suggesting that they may be potential diagnostic and prognostic biomarkers for MPM.

There are several limitations in this study. The results and conclusions obtained are based on microarray data, and there is a lack of validation with experimental data with clinical samples. The sample size in prognostic analysis is 
relatively small. Further biological experiments are needed to elucidate the mechanisms behind the expression changes of these key genes and their biological functions in MPM.

In conclusion, our analysis has identified a number of DEGs that are closely related to the development, progression, and prognosis of MPM. A total of 277 DEGs and 24 hub genes have been found this study. EZH2 and HMMR might be the core genes of MPM based on their high connectivity in the protein interaction network. These findings may provide clues to develop the potential biomarkers for diagnosis of MPM and insight on molecular mechanisms underlying MPM to identify novel pharmacological and therapeutic targets for the treatment of MPM. However, further experimental verifcation and clinical studies are needed to confrm the potential biological functions and prognositic significance for MPM.

\begin{abstract}
Abbreviaitons
MPM: Malignant pleural mesothelioma; GEO: Gene Expression Omnibus; DEG: Differentially expressed genes; PPI: Protein-protein interaction network; GO: Gene ontology; KEGG: Kyoto encyclopedia of genes and genomes; SETD2: SET domain containing protein 2; STRING: Search Tool for the Retrieval of Interacting Genes; MCODE: Molecular complex detection; MF: Molecular function; CC: Cellular component; BP: Biological process.
\end{abstract}

\section{Acknowledgements}

None.

\section{Authors' contributions}

$X L$ and $Y Z$ designed the study. $X L, K Q, G L$ and $P C$ collected the data and performed analysis. $\mathrm{XL}, \mathrm{KQ}$ and $\mathrm{YZ}$ drafted the manuscript. All authors read and approved the final manuscript.

\section{Funding}

This project was founded by Beijing Municipal Administration of Hospitals Clinical Medicine Development of Special Funding Support (Grant No. XMLX201702). The funding source had no involvement in study design; in the collection, analysis and interpretation of data; in the writing of the report; and in the decision to submit the article for publication.

\section{Availability of data and materials}

The datasets used and/or analysed during the current study are available from the corresponding author on reasonable request.

\section{Declarations}

Ethics approval and consent to participate

This work does not involve human or animal study.

\section{Consent for publication}

Not applicable.

\section{Competing interests}

The authors declare no competing interests.

Received: 23 November 2020 Accepted: 18 March 2021

Published online: 13 April 2021
References

1. Remon J, Lianes P, Martinez S, Velasco M, Querol R, Zanui M. Malignant mesothelioma: new insights into a rare disease. Cancer Treat Rev. 2013;39(6):584-91.

2. Leong SL, Zainudin R, Kazan-Allen L, Robinson BW. Asbestos in Asia. Respirology. 2015;20(4):548-55.

3. Hamaidia M, Gazon H, Hoyos C, Hoffmann GB, Louis R, Duysinx B, et al. Inhibition of EZH2 methyltransferase decreases immunoediting of mesothelioma cells by autologous macrophages through a PD-1-dependent mechanism. JCI Insight. 2019;4(18):66.

4. Suveg K, Putora PM, Berghmans T, Glatzer M, Kovac V, Cihoric N. Current efforts in research of pleural mesothelioma - an analysis of the ClinicalTrials.gov registry. Lung Cancer. 2018;124:12-8.

5. Bueno R, Stawiski EW, Goldstein LD, Durinck S, De Rienzo A, Modrusan Z, et al. Comprehensive genomic analysis of malignant pleural mesothelioma identifies recurrent mutations, gene fusions and splicing alterations. Nat Genet. 2016;48(4):407-16.

6. Ernst A, Schlattmann P, Waldfahrer F, Westhofen M. Laryngorhinootologie. 2017:96(8):519-21.

7. Gemignani F, Neri M, Bottari F, Barale R, Canessa PA, Canzian F, et al. Risk of malignant pleural mesothelioma and polymorphisms in genes involved in the genome stability and xenobiotics metabolism. Mutat Res. 2009;671(1-2):76-83.

8. Comertpay S, Pastorino S, Tanji M, Mezzapelle R, Strianese O, Napolitano $\mathrm{A}$, et al. Evaluation of clonal origin of malignant mesothelioma. J Transl Med. 2014;12:301.

9. Cedres S, Montero MA, Martinez P, Martinez A, Rodriguez-Freixinos V, Torrejon D, et al. Exploratory analysis of activation of PTEN-PI3K pathway and downstream proteins in malignant pleural mesothelioma (MPM). Lung Cancer. 2012;77(1):192-8.

10. Yamagishi T, Fujimoto N, Nishi H, Miyamoto Y, Hara N, Asano M, et al. Prognostic significance of the lymphocyte-to-monocyte ratio in patients with malignant pleural mesothelioma. Lung Cancer. 2015;90(1):111-7.

11. Suraokar MB, Nunez MI, Diao L, Chow CW, Kim D, Behrens C, et al. Expression profiling stratifies mesothelioma tumors and signifies deregulation of spindle checkpoint pathway and microtubule network with therapeutic implications. Ann Oncol. 2014;25(6):1184-92.

12. Irizarry RA, Bolstad BM, Collin F, Cope LM, Hobbs B, Speed TP. Summaries of Affymetrix GeneChip probe level data. Nucleic Acids Res. 2003;31(4):e15.

13. Kanehisa M, Goto S. KEGG: kyoto encyclopedia of genes and genomes. Nucleic Acids Res. 2000;28(1):27-30.

14. Kanehisa M, Sato Y, Kawashima M, Furumichi M, Tanabe M. KEGG as a reference resource for gene and protein annotation. Nucleic Acids Res. 2016;44(D1):D457-62.

15. Rhodes DR, Kalyana-Sundaram S, Mahavisno V, Varambally R, Yu J, Briggs BB, et al. Oncomine 3.0: genes, pathways, and networks in a collection of 18,000 cancer gene expression profiles. Neoplasia. 2007;9(2):166-80.

16. Chandrashekar DS, Bashel B, Balasubramanya SAH, Creighton CJ, Ponce-Rodriguez I, Chakravarthi B, et al. UALCAN: a portal for facilitating tumor subgroup gene expression and survival analyses. Neoplasia. 2017;19(8):649-58.

17. Ivanova AV, Goparaju CM, Ivanov SV, Nonaka D, Cruz C, Beck A, et al. Protumorigenic role of HAPLN1 and its IgV domain in malignant pleural mesothelioma. Clin Cancer Res. 2009;15(8):2602-11.

18. Kaarteenaho-Wiik R, Soini Y, Pollanen R, Paakko P, Kinnula VL. Over-expression of tenascin-C in malignant pleural mesothelioma. Histopathology. 2003;42(3):280-91.

19. Hylebos M, Van Camp G, van Meerbeeck JP, Op de Beeck K. The genetic landscape of malignant pleural mesothelioma: results from massively parallel sequencing. J Thorac Oncol. 2016;11(10):1615-26.

20. Ou SH, Moon J, Garland LL, Mack PC, Testa JR, Tsao AS, et al. SWOG S0722: phase II study of mTOR inhibitor everolimus (RAD001) in advanced malignant pleural mesothelioma (MPM). J Thorac Oncol. 2015;10(2):387-91.

21. Yoshimura M, Kinoshita Y, Hamasaki M, Matsumoto S, Hida T, Oda Y, et al. Highly expressed EZH2 in combination with BAP1 and MTAP loss, as detected by immunohistochemistry, is useful for differentiating malignant pleural mesothelioma from reactive mesothelial hyperplasia. Lung Cancer. 2019;130:187-93.

22. Griffiths S, Loveday C, Zachariou A, Behan LA, Chandler K, Cole T, et al. EED and EZH2 constitutive variants: a study to expand the Cohen-Gibson 
syndrome phenotype and contrast it with Weaver syndrome. Am J Med Genet A. 2019;179(4):588-94.

23. Gango A, Batai B, Varga M, Kapczar D, Papp G, Marschalko M, et al. Concomitant 1 p36 deletion and TNFRSF14 mutations in primary cutaneous follicle center lymphoma frequently expressing high levels of EZH2 protein. Virchows Arch. 2018;473(4):453-62.

24. LaFave LM, Beguelin W, Koche R, Teater M, Spitzer B, Chramiec A, et al. Loss of BAP1 function leads to EZH2-dependent transformation. Nat Med. 2015;21(11):1344-9.

25. Kemp CD, Rao M, Xi S, Inchauste $S$, Mani H, Fetsch P, et al. Polycomb repressor complex-2 is a novel target for mesothelioma therapy. Clin Cancer Res. 2012;18(1):77-90.
26. Bahrami SB, Tolg C, Peart T, Symonette C, Veiseh M, Umoh JU, et al. Receptor for hyaluronan mediated motility (RHAMM/HMMR) is a novel target for promoting subcutaneous adipogenesis. Integr Biol (Camb). 2017;9(3):223-37.

\section{Publisher's Note}

Springer Nature remains neutral with regard to jurisdictional claims in published maps and institutional affiliations.
Ready to submit your research? Choose BMC and benefit from:

- fast, convenient online submission

- thorough peer review by experienced researchers in your field

- rapid publication on acceptance

- support for research data, including large and complex data types

- gold Open Access which fosters wider collaboration and increased citations

- maximum visibility for your research: over $100 \mathrm{M}$ website views per year

At BMC, research is always in progress.

Learn more biomedcentral.com/submissions 